\title{
PERSPECTIVE
}

\section{Does human language limit translatability of clinical and preclinical addiction research?}

\author{
Harriet de Wit ${ }^{1}$, David H. Epstein ${ }^{2}$ and Kenzie L. Preston ${ }^{2}$ \\ Neuropsychopharmacology (2018) 43:1985-1988; https://doi.org/10.1038/s41386-018-0095-8
}

There is a gap between neuroscientific research and clinical practice in addiction-and some responsibility for that gap has been assigned to shortcomings of animal models (ref. [1]; also, more broadly, see ref. [2]). The gap has persisted despite improvements in animal modeling of addiction. For example, since the discovery that laboratory animals will self-administer most drugs that are liable to misuse by humans [3-5], investigators have increasingly recognized that core feature of addiction, such as compulsive or maladaptive use, cannot be modeled via passive, experimenter-controlled exposure to a drug [6]. The next step for animal models, now in progress, has been to place self-administration in the context of choices-choices guided by conflicting drives, probabilistic outcomes, or delayed outcomes [7]. These models have already advanced our understanding of addiction, demonstrating that compulsive drug use is based on biobehavioral processes shared across mammalian species. However, as the animal models become more refined, it is important to consider a fundamental species difference that stems from the uniquely human capacity to obtain information through language.

Perhaps the sheer obviousness of this species difference accounts for its not being mentioned, even indirectly, in recent reviews and commentaries about issues in animal modeling [8,9]. We think, however, that it is critical to recognize the potential influence of verbally communicated information in humans when thinking about the possibilities and limitations of new animal models and their interpretation. Clear thinking about the difference between language-guided and languageless learning is critical for translational behavioral research.

We will not attempt to resolve ancient questions about the relationship between language and abstract thinking. It seems safe to say that the two evolved together in humans [10]. In this commentary, when we refer to language in humans, we are referring to its role as a fast lane for intake of abstract knowledge. In the realm of drug use, examples might be: "smoking causes cancer"; "stimulants can keep you awake to study for school exams, but if you overuse them, you'll feel panicky"; and "cannabis has (such-and-such effects that can become enjoyable with experience), but you might lose your job if you use cannabis and have a urine test that week." Nonverbal animals may be capable of learning species-relevant analogs of those contingencies, but the absence of language ensures that the learning will be, at best, laborious: it occurs mainly through direct (or in rare cases observational) interactions with the environment and through the actual consequences of behavior.
Because recent critiques of animal models have also called for incorporation of social factors [1], it is worth noting here that language in humans evolved in tandem with culture, which encompasses social norms and laws, religious and moral feelings, group identity, and interpersonal connections. Social norms make people aware of potential negative consequences of drug use without having to experience them personally. Although animals, particularly those in the wild, probably have "cultures" to varying degrees, those cultures are limited by inability to transmit the rich bodies of information that humans transmit through spoken or written communication.

How does the presence of language in humans affect the validity of the animal models of drug taking? It is difficult to draw a clear line between face validity (sometimes dismissed as irrelevant to a model's usefulness) and construct validity (typically considered crucial, but hard to judge for models of addiction because addiction is insufficiently understood). The practical goal, for translational research, is predictive validity. Ultimately, the predictive validity of animal models of addiction will depend on improvements in face/construct validity, which involve numerous challenges. In this commentary, we take a first step: we examine the modeling issues raised by involvement of language in human drug use at every stage-initial use, regular use, development of (or avoidance of) problem use, cessation, and relapse.

An important question here is whether learning that was acquired through direct experience is functionally comparable to learning that was acquired verbally. Interestingly, Atlas et al. [11] have conducted a series of controlled studies addressing this question in the context of neural changes during fear conditioning. They found that "instructed knowledge" (i.e., information derived from verbal communication) led to updates in only a subset of the brain regions that were updated by direct experience. This raises the possibility that experientially induced behavior change may not follow the same rules as verbally induced behavior change.

Despite numerous commonalities in behavioral control by contingencies in humans and nonverbal species, we argue that the human ability to communicate through language adds a unique and often under-recognized limitation on the ability to develop valid translational studies. First, we discuss key differences between forms of communication in animals and humans. Then we discuss the ways that information acquired through language, rather than actual experience, can influence drug-use behaviors at two key stages: first use of a drug, and cessation. Throughout, we discuss how the human ability to communicate

\footnotetext{
'Department of Psychiatry and Behavioral Neuroscience, University of Chicago, Chicago, IL 60637, USA and ${ }^{2}$ Intramural Research Program, National Institute on Drug Abuse, Baltimore, MD, USA

Correspondence: Harriet de Wit (hdew@uchicago.edu)
}

Received: 27 December 2017 Revised: 10 May 2018 Accepted: 12 May 2018

Published online: 17 May 2018 
complicates translation from animal models of drug use to human addictive behaviors.

\section{HUMAN LANGUAGE VERSUS NONHUMAN-ANIMAL COMMUNICATION}

A key question is how exactly human language differs from forms of communication used by nonverbal animals. Linguists have grappled with this question for decades and have identified several key criteria for differentiation [12]. For our purpose, the key criterion is displacement, the human ability to talk about things that are not in the immediate environment because they are remote, abstract, or imaginary. In contrast, animal communication is controlled by the immediate context: animals communicate about stimuli that are currently present or currently being experienced. For example, rats exposed to predator odors release pheromones to communicate anxiety and distress, which can be relieved by a buffering olfactory relief signal from a conspecific [13]. Mice in the same room as conspecifics that have been subjected to inflammatory pain or withdrawal from morphine or alcohol develop "bystander" hyperalgesia through olfactory cues [14]. In studies of self-administration, rats self-administer cocaine more readily in the presence of conspecific that is also selfadministering cocaine [15], and prairie voles that are allowed to consume alcohol in the presence of conspecific adjust their intake according to the conspecific's intake [16]. Yet, in each of these cases, the "referent" of the communication between the animals is about what is occurring in the moment, not about past or future events.

\section{LANGUAGE AND INITIAL USE OF DRUGS}

Language plays an important role in initiation of drug use, including whether initiation occurs at all, and, if it does occur, how it is experienced.

The decision to use or not use is typically based on social communication: conformity, peer influence, religious beliefs, legal sanctions, and social and cultural traditions, all of which are transmitted through largely verbal means, such that they can be understood without having been experienced or witnessed. These influences may change with new information about perceived risks for certain drugs, or with changes in legal constraints. All of these factors can change the propensity to use a drug without having experienced the drug's effects. For example, awareness of the adverse consequences of drug use in an addicted family member, or publicity about a celebrity death by overdose, might influence the decision never to try a drug. A large body of research indicates that the initial decision to use is influenced by peers and by social factors [17, 18], and public-health workers have incorporated those findings into prevention efforts. Prevention approaches are often classroom-based, and typically focus on helping individuals develop the knowledge and attitudes needed to make good choices or change harmful behaviors. These approaches are verbally communicated and, of course, impossible to model in animals.

Language also influences the effects of a first use of drug, because people usually come to drug use with prior knowledge about what to expect-knowledge almost always acquired through spoken or written words. First uses in humans are undertaken with expectations about how the drug might make them feel, how long it will last, and whether it is likely to be harmful. As proposed by Becker [19], a notable example is the first use of hallucinogens or psychedelics. These drugs markedly alter cognition or sensory function, and without prior briefing their effects would be alarming to most individuals. When these drugs are used for religious rituals or in controlled studies, the users are carefully prepared with trained guides or highly structured rituals [20-22]. When they are used in settings without the control achieved in laboratory studies, their effects are highly variable and often unpleasant [19]. There is also a substantial literature on the role of expectancies in initiation of drug use [23, 24], further emphasizing the observation that prior information, usually obtained through spoken or written communication, influences the first use in humans.

In marked contrast, nonverbal animals have no prior information about the drugs that are administered in the laboratory or about the process of drug administration. During the first administration of the drug, the sensory experiences are novel and unexpected, which may introduce an aversive component to the memory of the experience. Of course, this is how nonverbal animals learn about new things-via experience, unmediated by other information. However, drugs may be a special case, because the first effects of addictive drugs can be markedly different from their later effects. For example, nicotine and opiates produce nausea and other unpleasant effects upon first use, and if people had no other information about the effects of the drug they would likely avoid it. Although nonverbal animals learn by experience that aversive effects give way to positive effects with repeated use, this process can be greatly expedited by prior knowledge communicated through language.

Once a drug is ingested for the first time, the capacity for language can further influence the experience. For example, prior expectances can give meaning to the physiological and sensory effects of a drug, which, in turn, influence future use. Psychedelic drugs are again a useful example here; humans often describe their effects in spiritual terms, with no clear counterpart in nonverbal animals. Furthermore, learning processes are likely to be involved in effectively labeling and communicating internal sensations. People using cannabis for the first time sometimes struggle to identify the novel sensations they experience; having a lexicon available to describe the feelings creates context and meaning [19]. It would be difficult to describe the interoceptive experiences obtained from drugs without language, and feeling "high" from a drug may be experienced as either positive or negative, depending on social influences and expectancies communicated by language. This is reflected, for example, in the use of the phrase "pleasant sick" among the standard clinical descriptors for effects of opioids [25]. Ultimately, the valence of these experiences is likely to contribute to further use of a drug.

These mixtures of positive and negative effects can be studied in laboratory animals-for example, by observing patterns of advance and retreat in rats traversing an alley apparatus to selfadminister cocaine [26]. However, the animals must learn about mixed effects in a kind of informational vacuum.

\section{LANGUAGE AND CESSATION OF DRUG USE}

The capacity for language in humans is critical during treatment and efforts to reduce or abstain from drug use. Efforts to abstain depend on knowledge and understanding about long-term and usually probabilistic consequences of drug use, which are extremely difficult to model in laboratory animals. In the most commonly used laboratory models, cessation occurs through extinction, punishment, or availability of alternative rewards, all of which involve immediate, experiential events.

Extinction in animals involves unreinforced responding for a previously reinforced operant, and it has no simple counterpart in the daily experience of human drug users: despite the now commonplace observation that the euphoric effects of many drugs may diminish over repeated uses, humans rarely undertake repeated instances of drug seeking that are entirely unreinforced. Interestingly, certain cessation treatments in humans are based on the knowledge that the drug will no longer be rewarding (as in the case of treatment with disulfiram or an opioid antagonist). Human users may perform an unreinforced response to "test" the 
new contingency, but they do not need to do so repeatedly (or even once) to learn it.

Punishment in animal models involves direct, immediate physical consequences of each instance of drug seeking $[27,28]$. In humans, punishment for drug use is not usually immediate upon drug seeking or taking; it is delayed by a duration that can range from hours to years, depending on the nature of the punishment (e.g., remorse, social consequences, or health consequences). Advance knowledge of the contingency may derive from having seen the experiences of others, but is mostly obtained through language.

Cessation via access to alternative rewards is perhaps the closest parallel between laboratory models and the experiences of human drug users. In both laboratory animals and humans, the availability of an attractive nondrug reward overrides the value of the drug. Again, however, the way this plays out in humans is intimately bound up with language. Some of the alternative rewards that are most effective in humans are long-term life benefits about which they are told. This also applies to the way humans evaluate those rewards in relation to drug-associated rewards and punishments: these are choices among "bundles" of proximal and distal outcomes [29, 30]. Even if nonverbal animals could integrate outcome information across time horizons longer than a few minutes (usually they cannot: see refs. [31, 32]), there may be no practical way for experimenters to give them the information. It is not known whether learning that occurs purely through individual experience (in the case of animals) is a serviceable model of learning that is verbally communicated and culturally buttressed. The two forms of learning may differ in long-term stability, resilience to perturbation, or other things that are relevant to outcome; they may also differ in terms of the genetic or phenotypic traits that predict individual differences in outcome.

\section{LANGUAGE AND SUSTAINED ABSTINENCE}

Verbal communication plays a critical role during sustained treatment. Behavioral treatment is often used even in combination with pharmacotherapies, and for some forms of addiction, the only effective treatments are "behavioral"-which, in practice, often means verbal. These treatments include cognitivebehavioral therapy, Acceptance and Commitment Therapy, motivational enhancement, and case management. Why do we rely on these approaches in humans? One reason is that treatment-seeking drug users tend to have issues related to poor social support and financial problems that exacerbate the difficulties of achieving and maintaining abstinence. These require language to address, and even the most sophisticated model in a nonverbal animal cannot offer information on how or for whom these approaches will be most effective.

Even a treatment approach that is translated directly from animal models can run into unexpected barriers associated with language. In rats, cue-induced drug craving can be reduced by behavioral or pharmacological interference with memory reconsolidation; in essence, this approach weakens or erases Pavlovian associations $[33,34]$. Our attempt to translate this to humans, using extinction trials for cocaine cues, was unsuccessful [35]. One reason for the failure may have been our participants' access to verbal information. Data published after we had finished our trial showed that memory-updating mechanisms are activated only when the outcome of an event is uncertain [36]. Our participants had signed consent forms describing the study, and the wording of the consent forms probably enabled participants to infer that we would never administer cocaine during the experimental sessions. Thus, there was no opportunity for memory reconsolidation because the participants knew too much-a problem that would not have occurred if we had been studying laboratory animals.

\section{CONCLUSION}

Despite all the issues we have raised here, animal models in addiction research have provided excellent insight into the neurobiology of drug use and addiction [37], and the developers of animal models have been exceedingly responsive to calls for models that incorporate choices and consequences [7, 38, 39]. The growing sophistication of those models is what led us to write this commentary bringing attention to the uniquely human capacity for language and its role in drug use, cessation, and treatment. The languageless nature of nonhuman animals may help explain why medications that looked promising in animal models have not translated into effective treatments in humans. We view this as a solvable puzzle: clearer thinking about the experiential differences of laboratory animals and human drug users should help guide the ongoing development of models of drug use and addiction, the ultimate goal of which-and the ultimate criterion for which-is their ability to identify effective new treatments.

\section{ACKNOWLEDGEMENTS}

This commentary was supported by DA02812 and DA037011 (to HdW) and by the Intramural Research Program of the National Institute on Drug Abuse, NIH (to DHE and KLP). Kasey Van Hedger, Scott Schepers, and Elisa Pabon provided helpful comments on the manuscript. HdW has received support unrelated to this manuscript from the following sources: consulting fees from Marinius and Jazz Pharmaceuticals; gift of a study drug from Indivior; and support for a research study from Insys Therapeutics.

\section{ADDITIONAL INFORMATION}

Competing interests: The authors declare no competing interests.

Publisher's note: Springer Nature remains neutral with regard to jurisdictional claims in published maps and institutional affiliations.

\section{REFERENCES}

1. Heilig M, Epstein DH, Nader MA, Shaham Y. Time to connect: bringing social context into addiction neuroscience. Nat Rev Neurosci. 2016;17:592-9.

2. Garner JP. The significance of meaning: why do over $90 \%$ of behavioral neuroscience results fail to translate to humans, and what can we do to fix it? ILAR J. 2014:55:438-56.

3. Fischman MW, Schuster CR. Drug seeking: a behavioral analysis in animals and humans. NIDA Res Monogr. 1978;20:4-23.

4. Thompson T, Schuster CR. Morphine self-administration, food-reinforced, and avoidance behaviors in rhesus monkeys. Psychopharmacologia. 1964;5:87-94.

5. Weeks JR. Experimental morphine addiction: method for automatic intravenous injections in unrestrained rats. Science. 1962;138:143-4.

6. Wise RA, Koob GF. The development and maintenance of drug addiction. Neuropsychopharmacology. 2014;39:254-62.

7. Ahmed SH. Validation crisis in animal models of drug addiction: beyond nondisordered drug use toward drug addiction. Neurosci Biobehav Rev. 2010;35:172-84.

8. Everitt BJ,Giuliano C,Belin D. Addictive behaviour in experimental animals: prospects for translation. Philos Trans R Soc Lond B Biol Sci. 2018;373:pii: 20170027

9. Kwako LE, Momenan R, Grodin EN, Litten RZ, Koob GF, Goldman D. Addictions neuroclinical assessment: a reverse translational approach. Neuropharmacology. 2017;122:254-64.

10. Deacon TW. The symbolic species: the co-evolution of language and the brain New York: Norton \& Company; 1997.

11. Atlas LY,Doll BB,Li J,Daw ND,Phelps EA. Instructed knowledge shapes feedbackdriven aversive learning in striatum and orbitofrontal cortex, but not the amygdala. Elife. 2016;5:pii: e15192

12. Hockett CF. The origin of speech. Sci Am. 1960;203:89-96.

13. Kiyokawa Y. Social odors: alarm pheromones and social buffering. Curr Top Behav Neurosci. 2017;30:47-65.

14. Smith $M L$, Hostetler $C M$, Heinricher MM, Ryabinin AE. Social transfer of pain in mice. Sci Adv. 2016;2:e1600855.

15. Smith MA, Zhang $H$, Robinson AM. The effects of excitatory and inhibitory social cues on cocaine-seeking behavior. Front Behav Neurosci. 2016;10:217.

16. Anacker AM, Loftis JM, Ryabinin AE. Alcohol intake in prairie voles is influenced by the drinking level of a peer. Alcohol Clin Exp Res. 2011;35:1884-90. 
17. Room R. Taking account of cultural and societal influences on substance use diagnoses and criteria. Addiction. 2006;101:31-9.

18. Stein JA, Newcomb MD, Bentler PM. An 8-year study of multiple influences on drug use and drug use consequences. J Pers Soc Psychol. 1987;53:1094-105.

19. Becker HS. History, culture and subjective experience: an exploration of the social bases of drug-induced experiences. J Health Soc Behav. 1967;8:163-76.

20. Griffiths RR, Johnson MW, Richards WA, Richards BD, Jesse R, MacLean KA, Barrett FS, Cosimano MP, Klinedinst MA. Psilocybin-occasioned mystical-type experience in combination with meditation and other spiritual practices produces enduring positive changes in psychological functioning and in trait measures of prosocial attitudes and behaviors. J Psychopharmacol. 2018;32:49-69.

21. Johnson M, Richards W, Griffiths R. Human hallucinogen research: guidelines for safety. J Psychopharmacol. 2008;22:603-20.

22. Kulis S, Hodge DR, Ayers SL, Brown EF, Marsiglia FF. Spirituality and religion: intertwined protective factors for substance use among urban American Indian youth. Am J Drug Alcohol Abus. 2012;38:444-9.

23. Chartier KG, Hesselbrock MN, Hesselbrock VM. Development and vulnerability factors in adolescent drug use. Child Adolesc Psychiatr Clin N Am. 2010;19:493-504.

24. Conway KP, Swendsen JD, Merikangas KR. Alcohol expectancies, alcohol consumption, and problem drinking: the moderating role of family history. Addict Behav. 2003;28:823-36

25. Fraser HF, Van Horn GD, Martin WR, Wolbach AB, Isbell H. Methods for evaluating addiction liability. (A) "Attitude" of opiate addicts toward opiate-like drugs. (B) a short-term "direct" addiction test. J Pharmacol Exp Ther. 1961;133:371-87.

26. Su ZI, Wenzel J, Baird R, Ettenberg A. Comparison of self-administration behavior and responsiveness to drug-paired cues in rats running an alley for intravenous heroin and cocaine. Psychopharmacology. 2011;214:769-78.

27. Panlilio LV, Thorndike EB, Schindler CW. Reinstatement of punishmentsuppressed opioid self-administration in rats: an alternative model of relapse to drug abuse. Psychopharmacology. 2003;168:229-35.
28. Pelloux Y, Everitt BJ, Dickinson A. Compulsive drug seeking by rats under punishment: effects of drug taking history. Psychopharmacology. 2007;194:127-37.

29. Heyman GM. Addiction and choice: theory and new data. Front Psychiatry. 2013;4:31.

30. Monterosso J, Ainslie G. The behavioral economics of will in recovery from addiction. Drug Alcohol Depend. 2007;90:S100-11.

31. Meck WH. Postreinforcement signal processing. J Exp Psychol Anim Behav Process. 1985;11:52-70.

32. Woolverton WL, Freeman KB, Myerson J, Green L. Suppression of cocaine selfadministration in monkeys: effects of delayed punishment. Psychopharmacology (Berl). 2012;220:509-17.

33. Luo YX, Xue YX, Liu JF, Shi HS, Jian M, Han Y, et al. A novel UCS memory retrievalextinction procedure to inhibit relapse to drug seeking. Nat Commun. 2015;6:7675.

34. Bernardi RE, Lattal KM, Berger SP. Postretrieval propranolol disrupts a cocaine conditioned place preference. Neuroreport. 2006;17:1443-7.

35. Jobes ML, Aharonovich E, Epstein DH, Phillips KA, Reamer D, Anderson M, Preston $\mathrm{KL}$. Effects of prereactivation propranolol on cocaine craving elicited by imagery script/cue sets in opioid-dependent polydrug users: a randomized study. J Addict Med. 2015:9:491-8.

36. Sevenster D, Beckers $T$, Kindt M. Retrieval per se is not sufficient to trigger reconsolidation of human fear memory. Neurobiol Learn Mem. 2012;97: 338-45.

37. Kalant $H$. What neurobiology cannot tell us about addiction. Addiction. 2010;105:780-9.

38. Venniro M, Caprioli D, Shaham Y. Animal models of drug relapse and craving: From drug priming-induced reinstatement to incubation of craving after voluntary abstinence. Prog Brain Res. 2016;224:25-52.

39. Deroche-Gamonet V, Belin D, Piazza PV. Evidence for addiction-like behavior in the rat. Science. 2004;305:1014-7. 